\title{
Hyperbolic QR factorization for $J$-spectral factorization of polynomial matrices
}

\author{
Didier Henrion ${ }^{1,2,3,4}$ Peter Hippe $^{5}$
}

July 12, 2004

\begin{abstract}
Motivated by a superfluous zero extraction problem arising in the discrete-time $J$-spectral factorization of polynomial matrices, we propose an algorithmic solution to hyperbolic QR factorization of a rank deficient constant matrix. Application to reduced-order $H_{\infty}$ filtering is illustrated by a numerical example.
\end{abstract}

\section{Keywords}

Polynomial matrices, $J$-spectral factorization, $H_{\infty}$ control, numerical linear algebra, computer-aided control system design.

\section{Introduction}

Polynomial methods offer an alternative to state-space methods when designing computeraided control system design algorithms. Amongst other software products, the Fortran SLICOT library [N98] incorporates mostly state-space methods, whereas the Polynomial Toolbox [P98] can be viewed as an alternative, or complementary product including various routines to deal with algebraic entities such as polynomials and polynomial matrices, based on the theory pioneered in [K79].

Keeping with our endeavour of designing efficient and reliable numerical routines for polynomial matrices for the Polynomial Toolbox package, in this paper we describe the

\footnotetext{
${ }^{1}$ Corresponding author. FAX: +33 5613369 69. E-mail: henrion@laas.fr

${ }^{2}$ LAAS-CNRS, 7 Avenue du Colonel Roche, 31077 Toulouse, France.

${ }^{3}$ Institute of Information Theory and Automation, Academy of Sciences of the Czech Republic, Pod vodárenskou věží 4, 18208 Praha, Czech Republic.

${ }^{4}$ Department of Control Engineering, Faculty of Electrical Engineering, Czech Technical University in Prague, Technická 2, 16627 Praha, Czech Republic.

${ }^{5}$ Universität Erlangen-Nürnberg, Lehrstuhl für Regelungstechnik, Cauerstrasse 7, D-91058 Erlangen, Germany.
} 
algorithmic solution of a hyperbolic QR factorization problem arising when pursuing a polynomial approach to discrete-time reduced-order $H_{\infty}$ filtering [HD02]. The problem concerns extraction of superfluous zeros when performing $J$-spectral factorization of polynomial matrices [KS94]. Spectral factorization is an operation that consists in extracting a solution of a quadratic polynomial matrix equation, and that can be viewed as a polynomial alternative to the state-space algebraic Riccati equation [K96].

In section 2 we briefly recall the polynomial solution to the discrete-time reduced-order $H_{\infty}$ filtering problem as described in [HD02]. We show that extraction of superfluous zeros is a necessary step when performing $J$-spectral factorization of polynomial matrices, and we describe formally in section 3 the corresponding hyperbolic QR factorization problem. An algorithmic solution is proposed in section 4, together with illustrative numerical examples. An application to the original $H_{\infty}$ filtering problem is also described. Numerical stability issues are mentioned in the conclusion.

\section{Reduced-order $H_{\infty}$ filtering}

Consider a time-invariant, discrete-time, linear system of order $n$ with unmeasurable outputs $y_{z}\left(\operatorname{size} p_{z}\right)$, measurements $y_{m}\left(\operatorname{size} p_{m}\right)$ and a vector of disturbances $w$ represented by

$$
y(z)=\bar{G}(z) w(z)=[G(s)+E] w(z)
$$

where $G(z)$ is the strictly proper part of $\bar{G}(z)$, with the partitioning

$$
y=\left[\begin{array}{l}
y_{z} \\
y_{1} \\
y_{2}
\end{array}\right]=\left[\begin{array}{c}
y_{z} \\
y_{m}
\end{array}\right]
$$

and

$$
E=\left[\begin{array}{c}
0 \\
E_{1} \\
0
\end{array}\right]=\left[\begin{array}{c}
0 \\
E_{m}
\end{array}\right]
$$

and the left coprime factorization

$$
G(z)=D^{-1}(z) N(z)
$$

Output $y_{m}$ is subdivided such that $y_{1}$ contains the $p_{m}-p$ disturbed measurements and $y_{2}$ the $p$ perfect ones. We assume that matrix $E_{1}$ has full row rank and $\operatorname{det} D(z)$ does not have zeros at $z=0$.

The problem considered in $H_{\infty}$ filtering is the following. Given system (1) and its $p_{m}$ measurements $y_{m}$, find an estimate $\hat{y}_{z}$ for the $p_{z}$ unmeasurable linear combinations $y_{z}$ of the state $x$ in the $H_{\infty}$-norm minimization sense, i.e. such that the (worst-case) performance measure

$$
\sup _{w}\left\|y_{z}-\hat{y}_{z}\right\|_{2} /\|w\|_{2}
$$

over all non-zero real square summable functions $w$ on the interval $[0, \infty[$ is less than a given upper bound $\gamma$. 
A state-space (time-domain) solution to this problem was presented in [HD01], whereas a polynomial (frequency-domain) solution was described in [HD02]. In this paper we will focus on the polynomial solution.

For the filter design, it is assumed that the factorization (2) is such that the polynomial part of matrix

$$
D^{p}(z)=D(z)\left[\begin{array}{cc}
I & 0 \\
0 & z^{-1} I_{p}
\end{array}\right]
$$

is row proper, i.e. its highest row degree coefficient matrix $G_{r}\left[D^{p}(z)\right]$ has full rank. This can always be ensured by appropriate left unimodular operations, see [W74].

The optimal filter has the transfer function

$$
\hat{y}(z)=\left[\begin{array}{ll}
I_{p z} & 0
\end{array}\right] \tilde{D}^{-1}(z)[\tilde{D}(z)-D(z)]\left[\begin{array}{c}
0 \\
y_{m}(z)
\end{array}\right]
$$

where $\tilde{D}(z)$ parametrizes the optimal $H_{\infty}$ filter. As explained in [HD01], in order to obtain the optimal filter matrix $\tilde{D}(z)$ we need as an intermediate result the parametrizing polynomial matrix $\tilde{D}_{f}(z)$ for the fictive filter (assuming $y_{z}$ to be measurable) described by

$$
\hat{y}_{f}(z)=\left[\begin{array}{ll}
I_{p z} & 0
\end{array}\right] \tilde{D}_{f}^{-1}(z)\left[\tilde{D}_{f}(z)-D(z)\right] y(z)
$$

with $\tilde{D}_{f}(z)$ and $D^{p}(z)$ sharing the same highest row degree coefficient matrices, i.e. $G_{r}\left[\tilde{D}_{f}(z)\right]=G_{r}\left[D^{p}(z)\right]$.

The polynomial matrix $\tilde{D}_{f}(z)$ is obtained via the following steps. First solve the $J$-spectral factorization equation

$$
\begin{gathered}
D_{f}(z) J D_{f}^{T}\left(z^{-1}\right)=D(z)\left[\begin{array}{cc}
-\gamma^{2} I_{p_{z}} & 0 \\
0 & E_{m} E_{m}^{T}
\end{array}\right] D^{T}\left(z^{-1}\right)+N(z) N^{T}\left(z^{-1}\right)+ \\
N(z) E^{T} D^{T}\left(z^{-1}\right)+D(z) E N^{T}\left(z^{-1}\right)
\end{gathered}
$$

The polynomial matrix $D_{f}(z)$ resulting from factorization (3) may contain $q$ superfluous zeros at $z=0$ which must be extracted by column operations

$$
D_{r}(z) V(z)=D_{f}(z)
$$

such that

$$
\operatorname{det} V(z)=z^{q}, \quad V(z) J V^{T}\left(z^{-1}\right)=J .
$$

This can be done by taking the zero coefficient matrix

$$
A=D_{f}^{T}(0)
$$

which due to the zeros of $D_{f}(z)$ at $z=0$ is rank deficient with $\operatorname{rank} A=n-q$, and finding a so-called hyperbolic QR factorization

$$
\begin{gathered}
A=Q R \\
Q^{T} J Q=J .
\end{gathered}
$$


Indeed, from factorization (5) matrix $D_{f}(0) Q^{T}=R^{T}$ has $q$ zero columns, and polynomial matrix $D_{f}(z) Q^{T}$ has a common factor $z$ in all non-zero elements of the corresponding columns that can be extracted by a matrix

$$
E(z)=\operatorname{diag}\left[\begin{array}{lllllll}
1 & \cdots & z^{-1} & \cdots & 1 & \cdots & z^{-1}
\end{array}\right]
$$

giving

$$
V^{-1}(z)=Q^{T} E(z)
$$

With the above $D_{r}(z)$, the parametrizing polynomial matrix for the fictive filter finally is

$$
\tilde{D}_{f}(z)=D_{r}(z) G_{r}^{-1}\left[D_{r}(z)\right] G_{r}\left[D^{p}(z)\right]
$$

To sum up, a key step in obtaining the $H_{\infty}$ filter is the extraction of superfluous zeros in polynomial matrix $D_{f}(z)$ via hyperbolic QR factorization (5).

\section{Problem statement}

The hyperbolic QR factorization problem arising in discrete-time $J$-spectral factorization of polynomial matrices can be formally stated as follows:

HQR Problem: Given an $n$-by- $n$ square matrix $A$ of rank $m$ and a signature matrix $J$, compute a full rank matrix $Q$ and a matrix $R$ of rank $m$ with $n-m$ zeros rows satisyfing relations (5).

Some remarks are in order.

Contrary to the classical QR factorization (corresponding to $J=I$ or $J=-I$ ), it is not assumed here that matrix $R$ in equation (5a) has an upper-triangular structure. For our $J$-spectral factorization application, the key property is that $R$ has just as many zero rows as the rank defect of $A$.

Matrix $Q$ in (5b) is referred to as a $J$-orthogonal matrix. Properties and generation of $J$-orthogonal matrices have been surveyed in [KSH00, App. B] and [H02]. Besides the $J$-spectral factorization problem studied here, $J$-orthogonal matrices arise in fast array algorithms for estimation and filtering [KSH00], as well as in the numerical solution of various matrix problems including downdating of Cholesky factorization, symmetric

definite generalized eigenvalue problems, study of $J$-contractive matrices, see [H02] and references therein.

\section{Algorithm}

In order to provide an algorithmic solution to problem HQR, we briefly recall properties and generation of orthogonal and hyperbolic rotation matrices. 


\subsection{Orthogonal rotations}

An orthogonal rotation (also called plane, or Givens rotation) zeroes a single element of a vector or matrix. Suppose we are given a vector

$$
a=\left[\begin{array}{l}
a_{1} \\
a_{2}
\end{array}\right]
$$

and we want to cancel entry $a_{2}$ with a row operation. Define the rotation matrix

$$
Q=\left[\begin{array}{cc}
c & s \\
-s & c
\end{array}\right], \quad c^{2}+s^{2}=1
$$

and notice that

$$
Q a=\left[\begin{array}{c}
\sqrt{a_{1}^{2}+a_{2}^{2}} \\
0
\end{array}\right]
$$

for the choice

$$
c=\frac{a_{1}}{\sqrt{a_{1}^{2}+a_{2}^{2}}}, \quad s=\frac{a_{2}}{\sqrt{a_{1}^{2}+a_{2}^{2}}} .
$$

Due to the constraint $c^{2}+s^{2}=1$, matrix $Q$ in equation (6) is orthogonal, i.e.

$$
Q^{T} Q=I \text {. }
$$

All the entries but one can be cancelled in a vector or matrix column by successive applications of the above 2-by-2 orthogonal rotations. This is the rationale behind QR factorization, where lower-triangular entries in a matrix are cancelled columnwise, producing an upper-triangular matrix $R$ and an orthogonal matrix $Q$ satisfying equation (5a), see [GV96] for details.

\subsection{Hyperbolic rotations}

Just like orthogonal rotations, hyperbolic rotations are used to cancel an individual entry in a vector or matrix. A hyperbolic rotation has the form

$$
Q=\left[\begin{array}{cc}
c & -s \\
-s & c
\end{array}\right], \quad c^{2}-s^{2}=1
$$

Its name origins from the fact that $c=\cosh \theta=\frac{1}{2}\left(e^{\theta}+e^{-\theta}\right)$ and $s=\sinh \theta=\frac{1}{2}\left(e^{\theta}-e^{-\theta}\right)$ for some $\theta$, compare with the plane rotation matrix (6) for which $c=\cos \theta$ and $s=\sin \theta$.

The equation

$$
Q a=\left[\begin{array}{l}
b \\
0
\end{array}\right]
$$

has a real solution only when $\left|a_{1}\right|>\left|a_{2}\right|$, in which case

$$
c=\frac{a_{1}}{\sqrt{a_{1}^{2}-a_{2}^{2}}}, \quad s=\frac{a_{2}}{\sqrt{a_{1}^{2}-a_{2}^{2}}}, \quad b=\sqrt{a_{1}^{2}-a_{2}^{2}} .
$$


It can be checked that matrix $Q$ is $J$-orthogonal, i.e.

$$
Q^{T} J Q=J
$$

for signature matrices

$$
J=\left[\begin{array}{cc}
1 & 0 \\
0 & -1
\end{array}\right] \quad \text { or } \quad\left[\begin{array}{cc}
-1 & 0 \\
0 & 1
\end{array}\right]
$$

Since a hyperbolic rotation can be applied only when $\left|a_{1}\right|>\left|a_{2}\right|$, it is necessary to carry out a row permutation prior to the transformation when $\left|a_{1}\right|<\left|a_{2}\right|$. As a result, unlike for orthogonal reductions, one cannot choose arbitrarily the locations of elements to be zeroed in a vector or matrix.

Finally, in the case $\left|a_{1}\right|=\left|a_{2}\right|$, no hyperbolic transformation can be applied. It means that, unlike for orthogonal reductions and the QR factorization, in general successive applications of hyperbolic rotations do not result in an upper-triangular matrix $R$.

\subsection{Algorithm description}

The algorithmic solution to problem HQR combines row and column orthogonal rotations, row hyperbolic rotations and row permutations. Basically, the algorithm mimics standard QR factorization: it consists in successive columnwise cancellations, until all linearly dependent rows appear at the bottom of the transformed matrix.

The main difference with standard QR factorization, which is also the main difficulty, concerns processing of the case when no row hyperbolic rotation can be applied. Below we will show that under the following assumption on input matrices $A$ and $J$, a hyperbolic rotation can always be applied.

Assumption: Matrices $A$ and $J$ satisfy

$$
\operatorname{rank} A^{T} J A=\operatorname{rank} A=m .
$$

Obviously, the above rank relation necessarily holds since if algorithm HQR successfully returns matrices $Q$ and $R$ satisfying relations (5), then

$$
A^{T} J A=R^{T} Q^{T} J Q R=R^{T} J R .
$$

Denoting by $U$ and $V$ the matrices obtained by keeping only the $m$ non-zero rows in matrices $R$ and $J R$, respectively, relation (7) follows from Sylvester's rank inequality applied on the product $U^{T} V$.

Conversely, suppose that at some step $k$ of the algorithm we obtain matrices $R_{k}$ and $Q_{k}$ such that

$$
Q_{k} A=R_{k}, \quad Q_{k}^{T} J Q_{k}=J
$$

and we are processing column $c_{1}$. Let $a_{1}$ in this column denote the leading entry in row $r_{1}$ in matrix $R_{k}$, used to cancel entry $a_{2}$ in row $r_{2}$ in the same column. Suppose that 
these entries correspond to different signs, so that a hyperbolic rotation must be applied. Moreover, suppose that $\left|a_{1}\right|=\left|a_{2}\right|$. Then several cases must be distinguished:

- either some remaining entries in the column have not yet been cancelled, in which case the algorithm proceeds to cancel remaining entries (see example 5.2 to understand why), and then the same column is processed again;

- or all the remaining entries in the column have been cancelled, in which case

- either rows $r_{1}$ and $r_{2}$ are linearly independent; then there exists a column $c_{2}$ with elements $b_{i}$ in the same row as $a_{i}$ for $i=1,2$, such that $a_{1} b_{2} \neq a_{2} b_{2}$. We can then combine columns $c_{1}$ and $c_{2}$ with the help of a column orthogonal rotation $U_{k}$ so that the resulting matrix $R_{k} U_{k}$ features leading entries $a_{1}$ and $a_{2}$ such that $\left|a_{1}\right| \neq\left|a_{2}\right|$. For a reason that should be clear with example 5.3, we choose a random $2 \times 2$ orthogonal rotation. Then the algorithm proceeds and the same column is processed again;

- or $r_{1}= \pm r_{2}$, so that rows $r_{1}$ and $r_{2}$ are linearly dependent. Then when evaluating the product

$$
A^{T} J A=R_{k}^{T} J R_{k}=S_{k}+r_{1}^{T} r_{1}-r_{2}^{T} r_{2}=S_{k}
$$

it follows that

$$
\operatorname{rank} A^{T} J A=\operatorname{rank} S_{k}=\operatorname{rank} A-1
$$

which contradicts our rank assumption (7). In other words, this situation never occurs.

The above analysis shows that the HQR problem can always be solved as soon as condition (7) is satisfied.

Based on these observations, a schematic description of Algorithm HQR is as follows.

Algorithm HQR: Proceed columnwise, denoting $a_{1}$ the pivot entry in row number $i_{1}$ and column number $j_{1}$ used to cancel iteratively bottom entries $a_{2}$ in rows number $i_{2}>i_{1}$ and column number $j_{1}$. If entry $a_{2}$ is non-zero and

- $J_{i_{1}}=J_{i_{2}}$ then cancel $a_{2}$ with an orthogonal rotation;

- $J_{i_{1}} \neq J_{i_{2}}$ and $\left|a_{1}\right| \neq\left|a_{2}\right|$ then if

- $\left|a_{1}\right|>\left|a_{2}\right|$ then cancel $a_{2}$ with a hyperbolic rotation;

- $\left|a_{2}\right|>\left|a_{1}\right|$ then permute rows $i_{1}$ and $i_{2}$ and cancel $a_{1}$ with a hyperbolic rotation;

- $J_{i_{1}} \neq J_{i_{2}}$ and $\left|a_{1}\right|=\left|a_{2}\right|$ then

- try first to cancel all remaining entries in column $j_{1}$;

- if all remaining entries in column $j_{1}$ have already been cancelled, then find column index $j_{2}$ such that $a_{1} b_{2} \neq a_{2} b_{2}$ where $b_{k}$ is the entry in row $i_{k}$ and column $j_{2}$. Combine columns $j_{1}$ and $j_{2}$ with an orthogonal rotation, and process again column $j_{1}$. 
The above algorithm has been implemented in a documented, stand-alone Matlab m-file available at

$$
\text { http://www.laas.fr/ henrion/software/hqr }
$$

This function will be included to the next release 3.0 of the commercial Polynomial Toolbox for Matlab, see [P98].

\section{$5 \quad$ Examples}

\subsection{No solution to problem HQR}

With this basic example we want to show that matrices $A$ and $J$ may exist for which assumption (7) is not satisfied. Just take

$$
A=\left[\begin{array}{ll}
1 & 0 \\
1 & 0
\end{array}\right], \quad J=\left[\begin{array}{cc}
1 & 0 \\
0 & -1
\end{array}\right] .
$$

It holds rank $A=1$ yet $\operatorname{rank} A^{T} J A=0$. As a result, problem HQR cannot be solved.

\subsection{Cancelling all remaining entries first}

This example shows that, when no hyperbolic rotation can be applied because $\left|a_{1}\right|=\left|a_{2}\right|$, then it is recommended first to process remaining entries in the column.

Let

$$
A=\left[\begin{array}{llll}
1 & 1 & 1 & 0 \\
0 & 0 & 1 & 0 \\
0 & 0 & 1 & 0 \\
0 & 0 & 1 & 0
\end{array}\right], \quad J=\left[\begin{array}{cccc}
1 & 0 & 0 & 0 \\
0 & -1 & 0 & 0 \\
0 & 0 & 1 & 0 \\
0 & 0 & 0 & -1
\end{array}\right] .
$$

Check that $\operatorname{rank} A^{T} J A=\operatorname{rank} A=2$, so assumption (7) is satisfied, and matrix $A$ can be reduced to the form (5).

When processing the third column with leading entry $a_{1}=A_{23}=1$ with sign $J_{2}=-1$, entry $a_{2}=A_{33}=1$ with opposite sign $J_{3}=1$ cannot be cancelled with a hyperbolic rotation. Yet next entry $a_{2}=A_{43}=1$ with sign $J_{4}=J_{2}=-1$ can be cancelled with an orthogonal rotation:

$$
Q_{1}=\left[\begin{array}{cccc}
1 & 0 & 0 & 0 \\
0 & \frac{\sqrt{2}}{2} & 0 & \frac{\sqrt{2}}{2} \\
0 & 0 & 1 & 0 \\
0 & -\frac{\sqrt{2}}{2} & 0 & \frac{\sqrt{2}}{2}
\end{array}\right], \quad R_{1}=\left[\begin{array}{cccc}
1 & 1 & 1 & 0 \\
0 & 0 & \sqrt{2} & 0 \\
0 & 0 & 1 & 0 \\
0 & 0 & 0 & 0
\end{array}\right], \quad Q_{1} A=R_{1} .
$$


Processing again the third column with the new leading entry $a_{1}=A_{23}=\sqrt{2}$, entry $a_{2}=A_{33}=1$ can be cancelled now with a hyperbolic rotation since $\left|a_{1}\right| \neq\left|a_{2}\right|$ :

$$
Q_{2}=\left[\begin{array}{cccc}
1 & 0 & 0 & 0 \\
0 & \sqrt{2} & -1 & 0 \\
0 & -1 & \sqrt{2} & 0 \\
0 & 0 & 0 & 1
\end{array}\right], \quad R_{2}=\left[\begin{array}{llll}
1 & 1 & 1 & 0 \\
0 & 0 & 1 & 0 \\
0 & 0 & 0 & 0 \\
0 & 0 & 0 & 0
\end{array}\right], \quad Q_{2} Q_{1} A=R_{2}
$$

Matrix $R_{2}$ is in the required form, and problem HQR is solved.

\subsection{Column orthogonal rotations}

In some special situations, all the remaining entries in a column have been cancelled, yet no hyperbolic rotation can be applied, and it may be necessary as a last resort to carry out a column orthogonal rotation. Such operations destroy the upper-triangular structure of matrix $R$, yet zero rows in $R$ are left unchanged.

Let

$$
A=\left[\begin{array}{lll}
1 & 1 & 1 \\
0 & 1 & 1 \\
1 & 0 & 0
\end{array}\right], \quad J=\left[\begin{array}{ccc}
1 & 0 & 0 \\
0 & -1 & 0 \\
0 & 0 & -1
\end{array}\right]
$$

It holds $\operatorname{rank} A^{T} J A=\operatorname{rank} A=2$, so assumption (7) is satisfied, and matrix $A$ can be reduced to the form $(5)$.

Processing the first column with leading entry $a_{1}=A_{11}=1$, no hyperbolic rotation can be used to cancel entry $a_{2}=A_{31}=1$, yet remaining entry $A_{21}=0$ is already zero. Since rows $r_{1}$ and $r_{3}$ are linearly independent, a column orthogonal rotation must be applied here. A natural choice would then be a permutation of columns 1 and 2:

$$
U_{1}=\left[\begin{array}{lll}
0 & 1 & 0 \\
1 & 0 & 0 \\
0 & 0 & 1
\end{array}\right], \quad R_{1}=\left[\begin{array}{lll}
1 & 1 & 1 \\
1 & 0 & 1 \\
0 & 1 & 0
\end{array}\right], \quad A U_{1}=R_{1}
$$

resulting in a matrix $R_{1}$ with $A_{31}=0$. However, the operation altered remaining entries in the column, and $a_{2}=A_{21}=1$ must now be cancelled with leading entry $a_{1}=A_{11}=1$. A hyperbolic rotation must be applied since $J_{1}=-J_{2}$, yet $\left|a_{1}\right|=\left|a_{2}\right|$. Remaining entry $A_{31}$ is zero, so that once more we must resort to a column orthogonal rotation. A natural choice would then be a permutation of column 1 and 2 :

$$
U_{2}=\left[\begin{array}{lll}
0 & 1 & 0 \\
1 & 0 & 0 \\
0 & 0 & 1
\end{array}\right], \quad R_{2}=\left[\begin{array}{lll}
1 & 1 & 1 \\
0 & 1 & 1 \\
1 & 0 & 0
\end{array}\right], \quad A U_{1} U_{2}=R_{2}
$$

However note that $R_{2}=A$, so that we entered a never-ending cycle.

For this reason, we propose the following way out: instead of applying a column orthogonal rotation to cancel entry $a_{2}$, we apply a random column orthogonal rotation, and then the 
column is processed again. In the above example, instead of permuting columns 1 and 2 , we combine them randomly, e.g.

$$
U_{1}=\left[\begin{array}{ccc}
0.9501 & 0.3119 & 0 \\
-0.3119 & 0.9501 & 0 \\
0 & 0 & 1
\end{array}\right], \quad R_{1}=\left[\begin{array}{ccc}
0.6383 & 1.2620 & 1 \\
-0.3119 & 0.9501 & 1 \\
0.9501 & 0.3119 & 0
\end{array}\right], \quad A U_{1}=R_{1} \text {. }
$$

Then we process again the first column with leading entry $a_{1}=A_{11}=0.6383$. Entry $A_{21}=-0.3119$ is first cancelled with a hyperbolic rotation:

$$
Q_{2}=\left[\begin{array}{ccc}
1.1461 & 0.5600 & 0 \\
0.5600 & 1.1461 & 0 \\
0 & 0 & 1
\end{array}\right], \quad R_{2}=\left[\begin{array}{ccc}
0.5569 & 1.9784 & 1.7061 \\
0 & 1.7957 & 1.7061 \\
0.9501 & 0.3119 & 0
\end{array}\right] \quad Q_{2} A U_{1}=R_{2}
$$

Proceeding this way, we eventually solve problem HQR with output matrices

$$
Q=\left[\begin{array}{ccc}
1.6393 & -0.4051 & -1.2342 \\
-1 & 1 & 1 \\
-0.8291 & -0.4051 & 1.2342
\end{array}\right], \quad R=\left[\begin{array}{ccc}
0.4051 & 1.2342 & 1.2342 \\
0 & 0 & 0 \\
0.4051 & -1.2342 & -1.2342
\end{array}\right]
$$

\section{$5.4 \quad H_{\infty}$ filtering}

Let system (1) be given by

$$
\begin{aligned}
y_{z}(z) & =\frac{1}{z^{2}-z+0.25}\left[\begin{array}{ccc}
z+3.25 & -3 z+1.5 & 0
\end{array}\right] w(z) \\
y_{m}(z) & =\left(\frac{1}{z^{2}-z+0.25}\left[\begin{array}{ccc}
2 z+0.25 & -z+0.5 & 0 \\
-z+3 & -2 z+1 & 0
\end{array}\right]+\left[\begin{array}{ccc}
0 & 0 & 1 \\
0 & 0 & 0
\end{array}\right]\right) w(z)
\end{aligned}
$$

so that $p_{z}=1, p_{m}=2$ and $p=1$. A left coprime factorization (2) of the strictly proper part

$$
\frac{1}{z^{2}-z+0.25}\left[\begin{array}{ccc}
z+3.25 & -3 z+1.5 & 0 \\
2 z+0.25 & -z+0.5 & 0 \\
-z+3 & -2 z+1 & 0
\end{array}\right]
$$

is given by

$$
N(z)=\left[\begin{array}{ccc}
0.7071 & -2.1213 & 0 \\
-1.4142 & -2.8284 & 0 \\
0 & 0 & 0
\end{array}\right]
$$

and

$$
D(z)=\left[\begin{array}{ccc}
0.4714 z-0.4125 & 0.2357 z-1.0017 & 0.2357 z+0.5893 \\
-0.4714 & -0.9428 & 1.4142 z+0.4714 \\
0.5774 & -0.5774 & -0.5774
\end{array}\right]
$$

With $\gamma=2.41523$ the right hand-side of polynomial matrix equation (3) reads

$$
\left[\begin{array}{ccc}
0.8982 z+3.7703+0.8982 z^{-1} & 1.0741 z+4.8102 & -1.7237 z+1.9675 \\
4.8102+1.0741 z^{-1} & 9.5926 & 2.1320 \\
1.9675-1.7237 z^{-1} & 2.1320 & -1.6111
\end{array}\right] \text {. }
$$


Applying $J$-spectral factorization with an updated version of macro spf (available upon request, and to be implemented in the next release of the Polynomial Toolbox for Matlab), the factorization result is

$D_{f}(z)=\left[\begin{array}{ccc}-0.5832 z+0.1117 & -0.9082 z-0.6920 & -1.6746 z-0.1221 \\ -0.3093 z+0.1336 & 0.5696 z-0.8276 & -2.9454 z-0.1460 \\ 2.0619 z-0.2145 & -0.1714 z+1.3281 & -0.9155 z+0.2343\end{array}\right], \quad J=\left[\begin{array}{ccc}-1 & 0 & 0 \\ 0 & 1 & 0 \\ 0 & 0 & 1\end{array}\right]$.

Polynomial matrix $D_{f}(z)$ has $n-p=1$ zero at $z=0.1429$ and $q=2$ zeros at $z=0$, which have to be extracted.

Applying algorithm HQR on the constant coefficient matrix $A=N_{f}^{T}(0)$ we obtain the extraction matrix

$$
V(z)=\left[\begin{array}{ccc}
-1.0129 z & 0.1586 z & 0.0280 z \\
-0.1611 & 0.9975 & 0.1760 \\
0 & 0.1738 z & -0.9848 z
\end{array}\right]
$$

such that the reduced matrix satisfying relation (4) reads

$$
D_{r}(z)=\left[\begin{array}{ccc}
0.7817 & -1.2946 z-0.6938 & 1.4913 \\
0.3054 & -0.8297 & 2.9996 \\
-2.0357 & 1.3315 & 0.8718
\end{array}\right]
$$

The above matrix has just one zero at $z=0.1429$, which corresponds to the pole of the fictive filter. The fictive filter is then obtained from polynomial matrix $D_{r}(z)$ along the lines given in [HD02], and we obtain

$$
\tilde{D}_{f}(z)=\left[\begin{array}{ccc}
0.4714 z-0.5135 & 0.2357 z-0.4125 & 0.8250 \\
-0.4714 & -0.9428 & 1.4142 \\
0.5774 & -0.5774 & 0
\end{array}\right]
$$

\section{Conclusion}

Hyperbolic QR factorization has been studied recently in reference [BHP03], from which most of the material found here was extracted. Note however than in [BHP03] the authors are concerned with solving an indefinite least squares problem for which matrix $A$ is rectangular and full column-rank, under the assumption that matrix $A^{T} J A$ is positive definite. In the development here, because of our polynomial $J$-spectral factorization background, matrix $A$ is assumed to be square (even though our results can be extended to rectangular matrices) but rank-deficient. Moreover, our algorithm works under the less stringent assumption that matrices $A^{T} J A$ and $A$ share the same rank. Finally, we are basically interested in zeroing rows in matrix $A$, without specific requirements on its triangular structure.

In [BHP03] it is mentioned that obtaining useful error bounds for the application of a product of hyperbolic transformations to a vector is much more difficult that when the transformations are orthogonal. It is well-known [GV96] that orthogonal rotations are backward stable, property on which numerically reliable algorithms such as the standard 
QR factorization heavily rely. This is in contrast with hyperbolic rotations, and it is presently unclear to numerical analysts whether the hyperbolic QR factorization algorithm is mixed backward-forward stable, or even backward stable. As recalled in [H02], eigenvalues and singular values of $J$-orthogonal matrices occur in reciprocal pairs and these matrices can be arbitrarily ill-conditioned. Hyperbolic rotations must thus be carried out in a careful way, as recommended in [KSH00, App. B] and [BHP03]. In order to minimize the number of operations, it is also possible to use Householder reflections, allowing to zero several entries at once in a vector. $J$-orthogonal Householder reflections are described in [KSH00, App. B].

\section{Acknowledgment}

This work was supported by the Grant Agency of the Czech Republic under Project No. 102/02/0709. We are grateful to Hans Grabmüller who largely contributed to this work.

\section{References}

[BHP03] A. Bojanczyk, N. J. Higham, H. Patel. Solving the indefinite least squares problem by hyperbolic QR factorization. SIAM Journal on Matrix Analysis and Applications, Vol. 24, No. 4, pp. 914-931, 2002.

[GV96] G. H. Golub, C. F. Van Loan. Matrix computations. 3rd edition. The Johns Hopkins University Press, New York, NY, 1996.

[H02] N. J. Higham. J-orthogonal matrices: properties and generation. SIAM Review, Vol. 45, No. 3, pp. 504-519, 2003.

[HD01] P. Hippe, J. Deutscher. Design of reduced order $H_{\infty}$ filters for discrete-time systems. Proceedings of the IFAC World Congress on Automatic Control, paper T-We-M18-1, Barcelona, Spain, 2002.

[HD02] P. Hippe, J. Deutscher. Frequency domain design of reduced order $H_{\infty}$ filters for discrete-time systems. Proceedings of the European Control Conference, Cambridge, UK, 2003.

[KSH00] T. Kailath, A. H. Sayed, B. Hassabi. Linear estimation. Prentice Hall, Upper Saddle River, NJ, 2000.

[K79] V. Kučera. Discrete Linear Control: The Polynomial Approach. John Wiley and Sons, Chichester, UK, 1979.

[KS94] H. Kwakernaak, M. Šebek. Polynomial $J$-spectral factorization. IEEE Transactions on Automatic Control, Vol. 39, No. 2, pp. 315-328, 1994.

[K96] H. Kwakernaak. Frequency domain solution of the standard $H_{\infty}$ problem. In M. J. Grimble, V. Kučera (Editors). Polynomial methods for control system design. Springer Verlag, Berlin, Germany, 1996. 
[N98] NICONET: The Numerics in Control Network. 1998-2002. See www. win.tue.nl/niconet

[P98] PolyX, Ltd. The Polynomial Toolbox for Matlab. Prague, Czech Republic. Version 2.5 released in 2000. See www. polyx.cz

[W74] W. A. Wolovich. Linear multivariable systems. Springer Verlag, New York, NY, 1974. 